\title{
Surveillance of abused drugs in forensic autopsy cases in Norway
}

\author{
Svetlana V. Konstantinova-Larsen, Per T. Normann, Marianne Arnestad, \\ Ritva Karinen, Asbjørg S. Christophersen and Jørg Mørland \\ Division of Forensic Medicine and Drug Abuse Research, Norwegian Institute of Public Health \\ Correspondence: Svetlana V. Konstantinova-Larsen, Division of Forensic Medicine and Drug Abuse Research, Norwegian Institute of Public \\ Health, P.O. Box 4404 Nydalen, NO-0403 Oslo, Norway \\ E-mail: svetlana.konstantinova@fhi.no Telephone: +47210779000 Telefax: +4721077878
}

\begin{abstract}
High drug related mortality has been registered in Norway. Although comparison between countries relies on a standard international coding system of diseases, different practices in verifying cause of death and applying codes could lead to variation. The comparison gives no information on drug findings or drug patterns underlying the cause of deaths. To evaluate deaths involving illicit drugs in Norway, we examined drug patterns in 2735 forensically examined post-mortem samples collected from 15-64 year-old individuals from 2000 to 2009 . There were four times as many men as women among the deceased illicit drug users, and the majority were in the age group 25-44 years. The number of deceased showing signs of illicit drug use has gradually declined during the study period. The decline was found among younger individuals, while a larger proportion of the deceased were above 45 years of age in 2009, compared to 2000. Cases positive for heroin, ethanol, ecstasy and flunitrazepam were fewer in 2009, while the prevalence of amphetamine, cannabis, methadone and other opioids has increased. The prevalence of methamphetamine has increased ten fold, and the prevalence of benzodiazepines doubled. Thus, the drug pattern and age of the deceased has changed markedly during the last 10 years. Heroin and ethanol use has partly been substituted by use of amphetamines, cannabis, benzodiazepines and other opioids. This change could possibly be explained by the prolonged survival of drug users on substitution treatment and by the reduced toxicity of consumed drugs.
\end{abstract}

\section{INTRODUCTION}

According to a report from the European Centre for Drugs and Drug Addiction (EMCDDA), Norway has high levels of drug related mortality compared to other countries in Europe (1). The comparison between countries relies on ICD-10 codes or police information on cause of death, as well as EMCDDA criteria for mortality related to drugs of abuse. Although standard criteria were used for the classification of death, comparisons between countries are difficult because coding practices, frequency of forensic examinations and autopsies, and analytical toxicological repertoires have not been standardized. Therefore, different drugs and drug combinations (drug patterns) could be included in different countries.

An earlier study has shown an increase in fatal drug poisonings among drug addicts in Norway from 1991 to 2007 (2). Drug findings were reported for all forensically examined overdoses in 1991, 1997, 2002 and 2007. The overdose mortality in that study was attributed to consumption of illicit drugs, medicinal drugs including opioids and benzodiazepines, as well as of ethanol (2,3). Misuse of illicit drugs had the highest effect on drug related mortality in young and middleaged individuals. Moreover, in 2007, the most prevalent form of intoxication was by multiple drug combinations. Compared to 1991 findings of heroin, cannabis and ethanol were less prevalent in 2007. However, findings of amphetamines, cocaine, metha- done and benzodiazepines became more frequent in more recent years.

The police (Kripos) reports on overdose mortality are not complete and limited in drug findings (4). Drug use assessed from self reports by the Norwegian Institute for Alcohol and Drug Research (SIRUS) may possibly be underestimated (4). Few epidemiological studies have assessed findings of drugs and drug patterns in post-mortem cases in Norway (2,5-7). As far as we know, trends in drug use in the general Norwegian population and changes in drug patterns over time have not previously been investigated in forensically examined deaths. The complete information on drug findings in deceased is collected from the forensic toxicological laboratory at the Norwegian Institute of Public Heath (NIPH). Monitoring drug findings in forensically examined autopsy cases from the general population was initiated in order to explore the drug use. The aim of our study was to describe trends of illicit drug use and drug patterns in 15-64 year-old fatalities who underwent forensic examination in Norway from 2000 to 2009.

\section{MATERIAL AND METHODS}

\section{Subjects}

The study sample was selected from a total of 16756 forensic autopsy cases examined at the Norwegian Institute of Public Health (NIPH) from the 1st of January 
Table 1. Total number of post mortem cases $(\mathrm{N}=16756)$ and cases positive for illicit drugs and/or methadone $(\mathrm{N}=2735)$ by gender and age in 2000-2009.

\begin{tabular}{lcccccccccc}
\hline Year & 2000 & 2001 & 2002 & 2003 & 2004 & 2005 & 2006 & 2007 & 2008 & 2009 \\
\hline Number of post-mortem cases $^{1}$ & 1855 & 1741 & 1768 & 1640 & 1645 & 1581 & 1579 & 1544 & 1659 & 1604 \\
N (\%) & $299(16)$ & $345(20)$ & $286(16)$ & $281(17)$ & $296(18)$ & $243(15)$ & $254(16)$ & $217(14)$ & $245(15)$ & $269(17)$ \\
N/1,000,000 & 103 & 118 & 97 & 95 & 99 & 81 & 83 & 70 & 78 & 85 \\
Males (\%) & 80 & 85 & 84 & 80 & 79 & 79 & 83 & 84 & 82 & 81 \\
Age (\%) & & & & & & & & & & 17 \\
$\quad 15-24 y$ & 19 & 21 & 17 & 16 & 20 & 21 & 19 & 17 & 13 \\
$25-34 y$ & 42 & 43 & 44 & 36 & 32 & 33 & 36 & 39 & 32 & 28 \\
$35-44 y$ & 29 & 26 & 26 & 28 & 32 & 28 & 27 & 25 & 31 & 25 \\
$45-54 y$ & 9 & 10 & 11 & 16 & 13 & 14 & 15 & 16 & 14 & 25 \\
$55-64 y$ & 1 & 1 & 2 & 4 & 4 & 3 & 3 & 3 & 6 & 9 \\
\hline
\end{tabular}

${ }^{1}$ Number of post-mortem cases forensically examined at NIPH.

${ }^{2}$ Number of drug positive cases $(\mathrm{N})$ per 1.000 .000 of 15-64 year-old population in Norway.

${ }^{3}$ Two sided $P$ value $<0.0001$ for the difference in age distribution of cases between years 2000 and 2009 .

2000 to the 31 st of December 2009 (Table 1). These cases comprised approximately $93-94 \%$ of toxicological examinations conducted in Norway during 20002009. The study sample was limited to 15-64 year-old individuals positive for one or several illicit drugs, such as heroin, amphetamines, cocaine and cannabis, or methadone. Thus, 2735 post-mortem cases were included in the study sample.

\section{Sampling and analyses of blood and urine}

Most of the samples (85\%) were collected during the first five days after death and $96 \%$ of the blood samples were taken from a peripheral (femoral, subclavian) vessel. Blood and urine were collected in $20 \mathrm{ml}$ Sterilin ${ }^{\circledR}$ tubes (Bibby Sterilin, Staffordshire, UK) containing $0.3 \mathrm{ml} 67 \%(\mathrm{w} / \mathrm{v})$ potassium fluoride solution and kept at $2-8^{\circ} \mathrm{C}$ from arrival in NIPH's toxicological laboratory until the analyses had been completed, normally within four weeks. The majority of cases underwent standardized analytical programmes. Blood and urine samples were examined for heroin markers including 6-monoacethylmorphine (6-MAM), morphine and codeine, as well as for amphetamine, methamphetamine and MDMA (ecstasy), cocaine, cannabis (tetrahydrocannabinol, THC), opioids other than heroin including methadone, buprenorphine, fentanyl, oxycodone, tramadol, pethidine, and for the benzodiazepines and Z-hypnotics including diazepam, nitrazepam, clonazepam, flunitrazepam, alprazolam, oxazepam, fenazepam, zolpidem, zopiclone, and ethanol.

All post-mortem blood and urine samples received by our laboratory were routinely screened for the drugs either by an immunological/enzymatic method or by chromatographic methods (8-10). The confirmation and quantification analyses for positive findings from screening were carried out using alternative chromatographic systems (11-13). Heroin exposure was assessed by the verification in both blood and urine of 6-MAM and/or a morphine to codeine concentration ratio greater than unity $(\mathrm{M} / \mathrm{C}>1)$, as suggested previously by us (14) and others (15).

\section{Statistical analysis}

Cases positive for 6-MAM in blood or urine and/or cases with $\mathrm{M} / \mathrm{C}>1$ in blood or urine were considered to be positive for heroin. The statistical analyses were performed for individual and grouped drugs. The amphetamine group included amphetamine, methamphetamine and ecstasy, the opioid group included methadone, buprenorphine, fentanyl, oxycodone, tramadol and pethidine, the benzodiazepine and Z-hypnotics group included diazepam, nitrazepam, clonazepam, flunitrazepam, alprazolam, oxazepam, fenazepam, zolpidem and zopiclone. The variable 'age' was categorised by five groups: 15-24, 25-34, 35-44, 45-54 and 55-64 years. Differences in demographic characteristics, drug findings and number of drugs per case between 2000 and 2009 were assessed by chi-squared tests. Difference in mean age between 2000 and 2009 was assessed by univariate analysis and adjusted for gender using multiple linear regression analysis. All statistical analyses were performed using SAS for Windows v9.2 (SAS Institute Inc., Cary, NC, USA).

\section{RESUlts}

\section{Characteristics of the study population}

Subject characteristics of the 2735 deceased positive for one or several illicit drugs, such as heroin, amphetamines, cocaine, cannabis, or methadone are shown in Table 1. The number of deceased per one million of the whole Norwegian population declined from 103 to 85 from 2000 to 2009 . The majority of the sample were men and the gender variation during the whole period was statistically non-significant. Deceased in the age group 25-44 years comprised $64 \%$ of the study group, while individuals younger or older than this were of equal proportions. There was no difference in the age distribution and mean age between men and women in the whole study population, or between the subgroups that died in 2000 and 2009. During the study period, the number of deceased in the age group 
15-44 years declined, while the number of 45-64 yearold individuals markedly increased $(P<0.0001)$. Median [mean $(95 \% \mathrm{CI})$ ] age was 32.0 [32.5 (31.5, 33.5)] in 2000 and 38.0 [(38.6 $(37.2,39.9)]$ in 2009 . In addition, multiple linear regression analysis adjusted for gender showed an increase in mean age of 6 years during the study period $(\mathrm{P}<0.0001)$.

\section{Illicit drugs and opioids}

Heroin was found in $54 \%$ of the study population, while psychostimulants (amphetamine, methamphetamine, MDMA and cocaine), cannabis and opioids were present in $40 \%, 39 \%$ and $22 \%$ of cases, respectively. In 2000, the prevalence of heroin was much higher than the prevalence of psychostimulants and cannabis, and findings of opioids were rare (Figure 1). From 2000 to 2009 , the proportion of heroin positive cases declined about $50 \%$ (from $74 \%$ to $38 \%$ ), while the proportion of psychostimulants, cannabis and opioids substantially increased. Overall, the prevalence of heroin, psychostimulants and cannabis findings were almost similar levels in 2009, 38\%, 48\% and 48\%, respectively.

Amphetamine was the most prevalent drug (35\%), compared to other drugs in the group of psychostimulants. Methamphetamine, ecstasy and cocaine were present in $19 \%, 3 \%$ and $2 \%$ of cases, respectively. During the study period, the prevalence of amphetamine and methamphetamine gradually increased from $25 \%$ to $36 \%$ and from $1 \%$ to $40 \%$, respectively (Figure 2 ).

Methadone was the most common drug in the opioids group (18\%). Buprenorphine, fentanyl, oxycodone and tramadol were found in $6 \%, 4 \%, 2 \%$ and $1 \%$ of cases, respectively. Only a few cases were positive for methadone or other opioids in 2000 (Figure 3). From 2000 to 2009, the prevalence of all opioids substantially increased. Moreover, in many cases positive for opioids, one or several other illicit drugs were also found. The proportion of these multi-drug cases increased from $50 \%$ to $72 \%$ during the study period.

\section{Benzodiazepines and ethanol}

Ethanol was found in $42 \%$ of drug users, while benzodiazepines and hypnotics were present in $66 \%$ of the study population. The proportion of drug users positive for ethanol was materially larger than the proportion of those consuming benzodiazepines and hypnotics in $2000,51 \%$ vs. $28 \%$, respectively (Figure 4 ), whereas the prevalence of benzodiazepines and hypnotics was approximately twice as high as the prevalence of ethanol in 2009.

Diazepam and flunitrazepam were the most frequently found drugs in the benzodiazepines and hypnotics group. They were found in $39 \%$ and $25 \%$ of cases, respectively. Prevalence of other drugs in the benzodiazepines group was $17 \%$ for clonazepam, $13 \%$ for nitrazepam, $12 \%$ for alprazolam, 12\% for oxazepam, $6 \%$ for zopiclone, $1 \%$ for zolpidem and $1 \%$ for fenazepam. Compared to 2000, significantly larger proportions of diazepam $(P<0.001)$, clonazepam $(P<$

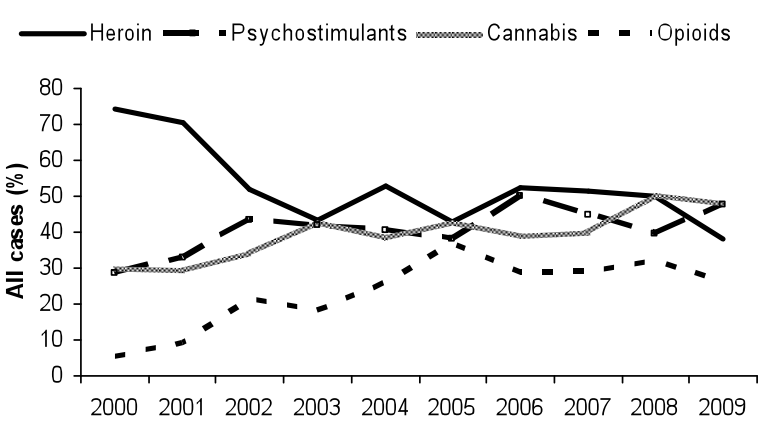

Figure 1. Prevalence of illicit drugs findings in 15-64 year-old drug users ${ }^{1,2,3}$.

${ }^{1}$ Psychostimulants: amphetamine, methamphetamine, MDMA (ecstasy), cocaine.

${ }^{2}$ Opioids other than heroin: methadone, buprenorphine, fentanyl, oxycodone, tramadol, pethidine.

${ }^{3}$ Two sided $P$ value $<0.0001$ for the difference in drug findings between years 2000 and 2009.

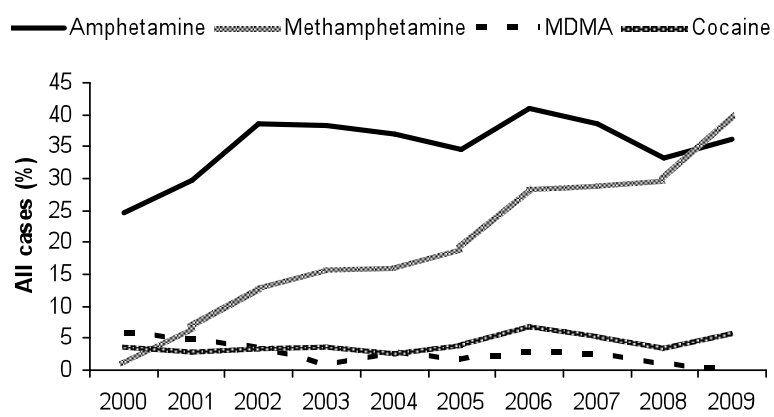

Figure 2. Prevalence of amphetamines and cocaine findings in 15-64 year-old drug users ${ }^{1}$.

${ }^{1}$ Two sided $P<0.001$ for the difference in amphetamine, methamphetamine and MDMA findings between years 2000 and 2009 , and $P>0.05$ for cocaine.

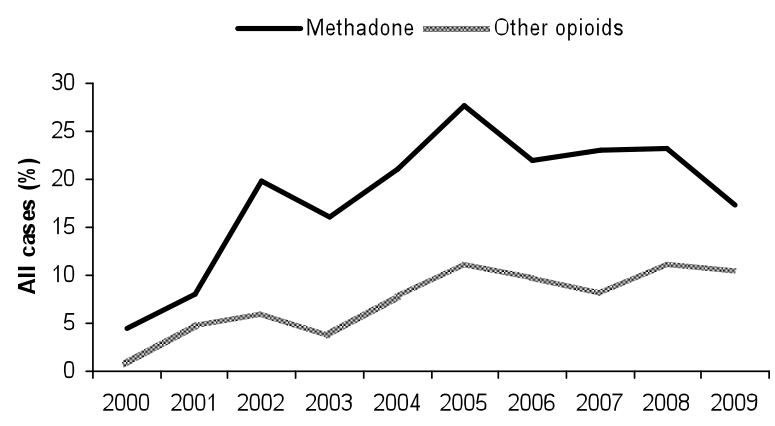

Figure 3. Prevalence of findings of opioids in 15-64 yearold drug users ${ }^{1,2}$.

${ }^{1}$ Opioids other than heroin: methadone, buprenorphine, fentanyl, oxycodone, tramadol, pethidine.

${ }^{2}$ Two sided $P$ value $<0.0001$ for the difference in drug findings between years 2000 and 2009.

$0.0001)$, oxazepam $(P<0.0001)$, nitrazepam $(P<$ 0.01), alprazolam $(P<0.0001)$, zopiclone $(P<0.0001)$ and zolpidem $(P<0.05)$ were found in 2009 . The proportion of flunitrazepam declined substantially $(P<$ 0.01 ) during the study period. 


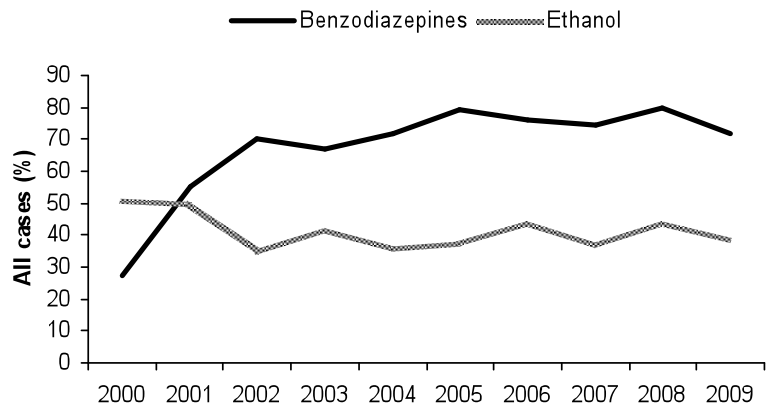

Figure 4. Prevalence of benzodiazepines and ethanol findings in 15-64 year-old users of illicit drugs ${ }^{1,2}$.

${ }^{1}$ Benzodiazepnes and Z-hypnotics: diazepam, nitrazepam, clonazepam, flunitrazepam, alprazolam, oxazepam, fenazepam, zolpidem, zopiclone.

${ }^{2}$ Two sided $P<0.001$ for the difference in drug findings between years 2000 and 2009.

$0.0001)$, oxazepam $(P<0.0001)$, nitrazepam $(P<$ 0.01), alprazolam $(P<0.0001)$, zopiclone $(P<0.0001)$ and zolpidem $(P<0.05)$ were found in 2009 . The proportion of flunitrazepam declined substantially $(P<$ 0.01 ) during the study period.

\section{Polydrug use}

The majority ( $66 \%$ ) of the deceased in 2000 were positive for one or two drugs, while fewer individuals were positive for three drugs or more (Figure 5). By contrast, in 2009 the proportion of cases positive for one or two drugs was lower $(33 \%)$, and those with more than two drugs had doubled.

\section{DisCUSSION}

This study showed that the prevalence of illicit drugs in forensic autopsy cases in Norway had declined from 2000 to 2009. The reduction was observed in 15-44 year-old men and women. Heroin, amphetamine, cannabis, ethanol and diazepam were the most frequently found drugs in the deceased, but, during the study pe- riod, the pattern of drug findings changed substantially. The prevalence of polydrug use doubled. Heroin, ethanol and flunitrazepam became less common, while drug combinations with amphetamine, methamphetamine, cannabis, diazepam, clonazepam and methadone became more prevalent by 2009. During the study period, the profile of deaths changed markedly from young individuals mainly consuming heroin alone or in a combination with ethanol, towards older individuals mainly misusing several drugs in combination.

One Nordic study has previously examined illicit drugs in overdose mortality in Norway in 2002 and 2007 (2). In this study, the number of deceased who tested positive for selected drugs declined from 2002 to 2007. A similar finding was observed in our study. In our study, the observed reduction of drug positive deaths from 2000 to 2009 was in line with a $10 \%$ decline in forensic autopsies during the same period.

Our results confirmed earlier findings that drug positive deaths are mostly in men aged 25-44 years (2). Our findings also demonstrated an increase in age for both men and women from 2000 to 2009 . The increase in age may be expected because of the increase in programmes supporting drug addicts and the fact that more addicts are enrolled in opioid maintenance treatment in Norway. Several studies on methadone maintenance treatment in opioid addicts demonstrated an increase of life expectancy for this population (16). The onset of polydrug use in older age can, however, not be excluded.

Our finding that approximately half the deaths involving illicit drug use relate to heroin consumption is in agreement with previously published reports (2). The observed decline from 2002 to 2009 could, however, partly be accounted for by the decline of forensic examinations during the period. Compared to heroin related deaths, deaths involving other illicit drugs became more common in 2009. A similar drug pattern has previously been observed in fatal poisonings of drug addicts (2). However, in this study the prevalence of

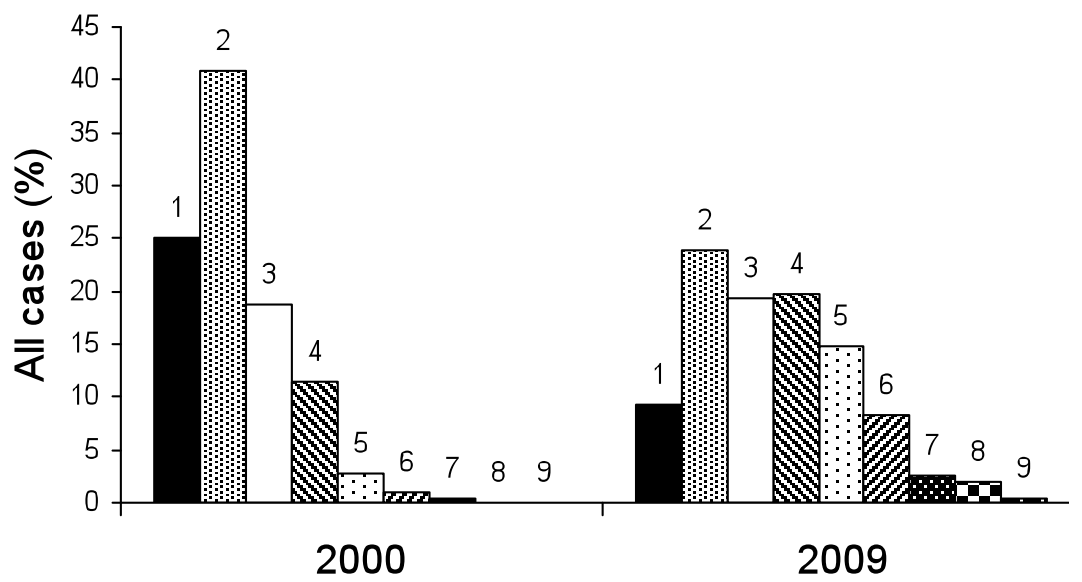

Figure 5. Number of drugs per case in 15-64 year-old drug users in 2000 and $2009^{1,2}$.

${ }^{1}$ Number of drugs per case: '1' indicates one drug, '2' indicates two drugs and respectively.

${ }^{2}$ Two-sided $P$ values $<0,0001$ for difference in number of drugs per case in 2009, compared to 2000. 
deceased who tested positive for amphetamines has substantially increased from 2000 to 2009 , and was almost at a similar level to heroin positive cases by 2009 despite of the decline in forensic examinations overall. This observation has not previously been reported in post-mortem cases. In addition, cases positive for other opioids, including methadone, became more frequent in 2009. Findings of illicit drugs in the majority of opioid positive cases could support our assumption about misuse of opioids and medicinal drugs by drug addicts.

The majority of deceased drug users combined illicit drugs with ethanol and/or benzodiazepines, and this was also the case for fatal poisonings (2). In our study, ethanol was the most used additional drug in 20002001, while benzodiazepines became the most frequently found drugs after 2001. The change in drug pattern could probably be accounted for by the change in preferences of drug users or/and the availability of benzodiazepines on the illegal marked.

Our findings are in agreement with earlier reports which show polydrug use in deceased drug addicts (2). The increase in the number of polydrug positive cases from 2000 to 2009 could probably be explained by misuse of both illicit drugs and the medicinal drugs available through drug replacement therapy. However, we cannot exclude the possibility of new polydrug mixtures being available on the illegal market. Reduced toxicity of drug combinations could probably reduce overdose mortality (17). Thus, changed drug patterns could reflect prolonged survival periods for illicit drug users. In this context, our findings suggest that the combined consumption of opioids and amphetamines could possibly reduce overdose mortality. However, this assumption needs more elaboration in future studies.

\section{Conclusion}

This study shows a modification in the profile of deceased individuals positive for illicit drugs in Norway from 2000 to 2009 . The age of the deceased and drug patterns changed over the study period. Abstinence from heroin and ethanol use, while consuming psychostimulants, cannabis, benzodiazepines and opioids is associated with older age of the deceased. Moreover, polydrug use became more common last years.

\section{REFERENCES}

1. European Monitoring Center for Drugs and Drug Addiction. The state of the drugs problem in Europe. In Annual report. EMCDDA, 2010.

2. Simonsen KW, Normann PT, Ceder G, Vuori E, Thordardottir S, Thelander G, et al. Fatal poisoning in drug addicts in the Nordic countries in 2007. Forensic Sci Int 207: 170-176 (2011).

3. Winther RB, Bramness JG. [Prescription shopping of addictive drugs in Norway]. Tidsskr Nor Laegeforen 129: $517-520$ (2009).

4. Norwegian Institute for Alcohol and Drug Research. Alcohol and drugs in Norway. In Annual report. EdlandGryt M, Ed. SIRUS, 2010.

5. Gjerde H, Christophersen AS, Normann PT, Mørland J. Toxicological investigations of drivers killed in road traffic accidents in Norway during 2006-2008. Forensic Sci Int 212: 102-109 (2011).

6. Gjerde H, Beylich KM, Mørland J. Incidence of alcohol and drugs in fatally injured car drivers in Norway. Accid Anal Prev 25: 479-483 (1993).

7. Mørland J, Steentoft A, Simonsen KW, Ojanpera I, Vuori E, Magnusdottir K, et al. Drugs related to motor vehicle crashes in northern European countries: A study of fatally injured drivers. Accid. Anal Prev 43: 19201926 (2011).

8. Gjerde H, Christophersen AS, Skuterud B, Klemetsen K, Mørland J. Screening for drugs in forensic blood samples using EMIT urine assays. Forensic Sci Int 44: 179-185 (1990).

9. Øiestad EL, Johansen U, Øiestad ÅML, Christophersen AS. Drug screening of whole blood by ultraperformance liquid chromatography-tandem mass spectrometry. J Anal Toxicol 35: 280-293 (2011).

10. Kristoffersen L Smith-Kielland A. An automated alcohol dehydrogenase method for ethanol quantification in urine and whole blood. J Anal Toxicol 29: 387-389 (2005).

11. Berg T, Lundanes E, Christophersen AS, Strand DH. Determination of opiates and cocaine in urine by high $\mathrm{pH}$ mobile phase reversed phase UPLC-MS/MS. J Chromatogr B Analyt Technol Biomed Life Sci 877: 421432 (2009).

12. Karinen R, Andersen JM, Ripel A, Hasvold I, Hopen AB, Mørland J, Christophersen AS. Determination of heroin and its main metabolites in small sample volumes of whole blood and brain tissue by reversed-phase liquid chromatography-tandem mass spectrometry. J Anal Toxicol 33: 345-350 (2009).

13. Sauve E, Øiestad ÅM, Middelkoop G, Krogh M, Ekeberg D. Determination of benzodiazepines in whole blood by ultra performance liquid chromatography tandem mass spectrometry. Ann Toxicol Anal 20 (Suppl 1): 77 (2008). 
14. Konstantinova SV, Normann PT, Arnestad M, Karinen R, Christophersen AS, Mørland J. Morphine to codeine concentration ratio in blood and urine as a marker of illicit heroin use in forensic autopsy samples. Forensic Sci Int (In press).

15. Jones AW, Holmgren A. Concentration ratios of free-morphine to free-codeine in femoral blood in heroinrelated poisoning deaths. Leg Med (Tokyo) 13: 171-173 (2011).

16. Esteban J, Gimeno C, Barril J, Aragones A, Climent JM, de la Cruz PM. Survival study of opioid addicts in relation to its adherence to methadone maintenance treatment. Drug Alcohol Depend 70: 193-200 (2003).

17. Trujillo AM, Smith ML, Guaderrama MM. Powerful behavioral interactions between methamphetamine and morphine. Pharmacol Biochem Behav 99: 451-458 (2011). 